\title{
Fine mapping of Noonan/cardio-facio cutaneous syndrome in a large family
}

\author{
E ric L egius, E Is Schollen, G ert M atthijs and Jean-Pierre Fryns \\ Center for H uman G enetics, Herestraat 49, 3000 L euven, B el gium
}

\begin{abstract}
Noonan syndrome (NS) is an autosomal dominant condition with facial dysmorphy, congenital cardiac defects and short stature. A gene for NS has previously been linked to a $14 \mathrm{cM}$ region in $12 \mathrm{q} 24 .{ }^{2}$ We performed linkage analysis in a four generation Belgian family with NS in some individuals and cardio-facio-cutaneous (CFC) syndrome in others. Clinical data and linkage data in this family indicate that NS and CFC syndrome result from a variable expression of the same genetic defect. We report a maximum lod score of 4.43 at zero recombination for marker D12S84 in 12q24. A crossover in this pedigree narrows the candidate gene region for $\mathrm{NS}$ to a $5 \mathrm{cM}$ interval between markers D12S84 and D12S1341.
\end{abstract}

Keywords: Noonan syndrome; cardio-facio cutaneous syndrome; CFC syndrome; linkage analysis; chromosome 12

\section{Introduction}

Noonan syndrome (NS) is an autosomal dominant condition characterised by a congenital heart defect, typical facial dysmorphy and short stature. ${ }^{1}$ Linkage of NS to chromosome 12 markers has been reported previously. A pproximately $50 \%$ of cases are sporadic, and only one large family is reported in the literature with proven linkage to $12 \mathrm{q} 24 .^{2}$ Some smaller nuclear families did not show linkage to this region, indicating genetic heterogeneity. In small families with NS and without proven linkage to 12q24 markers, crossovers cannot be interpreted, and therefore analysing large families is very important. Cardio-facio-cutaneous syndrome (CFC) is also characterised by heart defects, facial dysmorphy and short stature. A dditional features however are present such as skin abnormalities (hyperkeratosis, ichthyosis), hypotrichosis, retinal abnormalities and a moderate mental retardation or devel-

Correspondence: E ric Legius, Center for Human Genetics, H erestraat 49, 3000 L euven, B elgium

R eceived 17 M arch 1997; revised 28 A ugust 1997; accepted 3 September 1997 opmental delay. ${ }^{3}$ M ost CFC cases are sporadic. There has been a lot of debate in the literature regarding NS and CFC syndrome. Some clinicians favour the hypothesis that NS and CFC syndrome are two genetically different conditions. ${ }^{4} \mathrm{O}$ ther papers suggest that CFC syndrome and NS are allelic variants, ${ }^{5}$ or contiguous gene syndromes. ${ }^{6,7}$

We performed linkage analysis in a very large fourgeneration family, previously reported as an example of CFC syndrome ${ }^{8}$ to investigate possible linkage to $12 q 24$ markers.

\section{Subjects, Materials and Methods}

\section{Subjects}

We sampled 10 affected individuals, seven unaffected relatives, and three spouses from a large Belgian family after informed consent was obtained (Figure 1). Several individuals in this pedigree have been published previously. ${ }^{8} \mathrm{~A}$ II affected individuals have facial dysmorphy and short stature as in Noonan syndrome, and mild to moderate mental retardation. Individuals 1117 and 11112 are more retarded than their siblings and have IQs between 50 and 60 . III12 lives in an institution for mentally retarded adults and he has a mild valvular pulmonic stenosis. Individual III 2 was operated on 


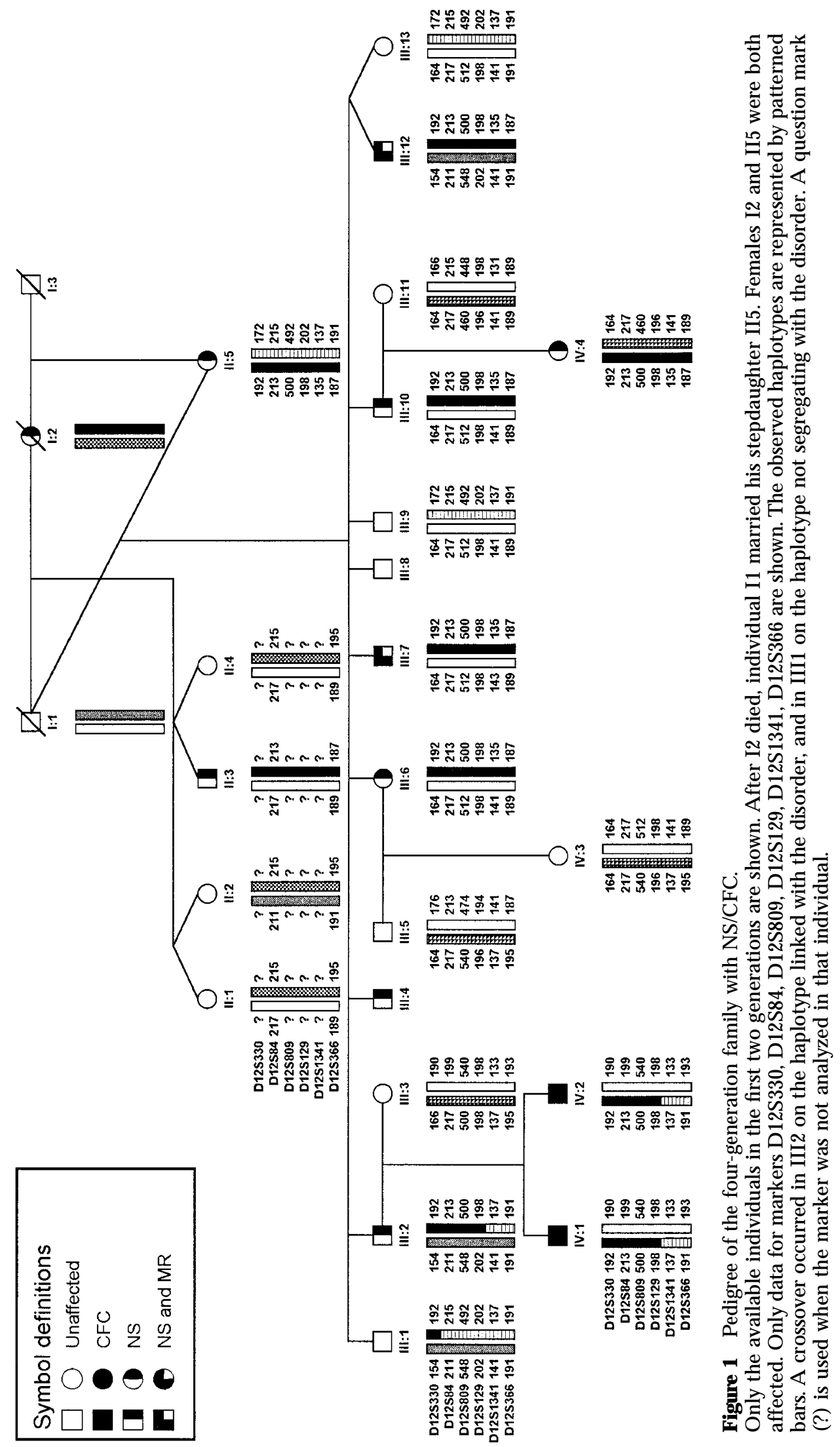


for a large ventricular septal defect and he received a valvulotomy for a pulmonic stenosis. $\mathrm{H}$ is two sons IV $\mathrm{I}$ and IV 2 are also mildly retarded and their phenotype was compatible with the CFC syndrome. They have the same facial dysmorphy and growth retardation as in N S but show in addition a dry scaly skin with hyperkeratotic palms, thin sparse scalp hair and scanty eyebrows (Figures 2 and 3). $O$ ther affected individuals have a phenotype more typical of NS and are less severely affected. ${ }^{8}$ Individual IV 4 underwent balloon dilatation for severe pulmonic stenosis with a dysplastic valve.

\section{Genotyping and Linkage Analysis}

G enomic D NA was extracted from peripheral blood lymphocytes by standard procedures. Twelve dinucleotide repeat markers in the $12 q 24$ region were PCR amplified from genomic DNA (Table 1). Of each PCR primer pair, one primer is fluorescently labelled (FITC, fluorescein-isothiocyanate). The resulting PCR fragments were sized on a Long $R$ anger $^{T M}$ gel in an ALF DNA Sequencer ${ }^{T M}$ (Pharmacia Biotech, U ppsala, Sweden) using the A LF Fragment Manager $^{\text {TM }} 1.0$ software (Pharmacia Biotech, U ppsala, Sweden). A llele sizes were assigned by comparison with a fluorescently labelled DNA molecular-size standard and with the CEPH

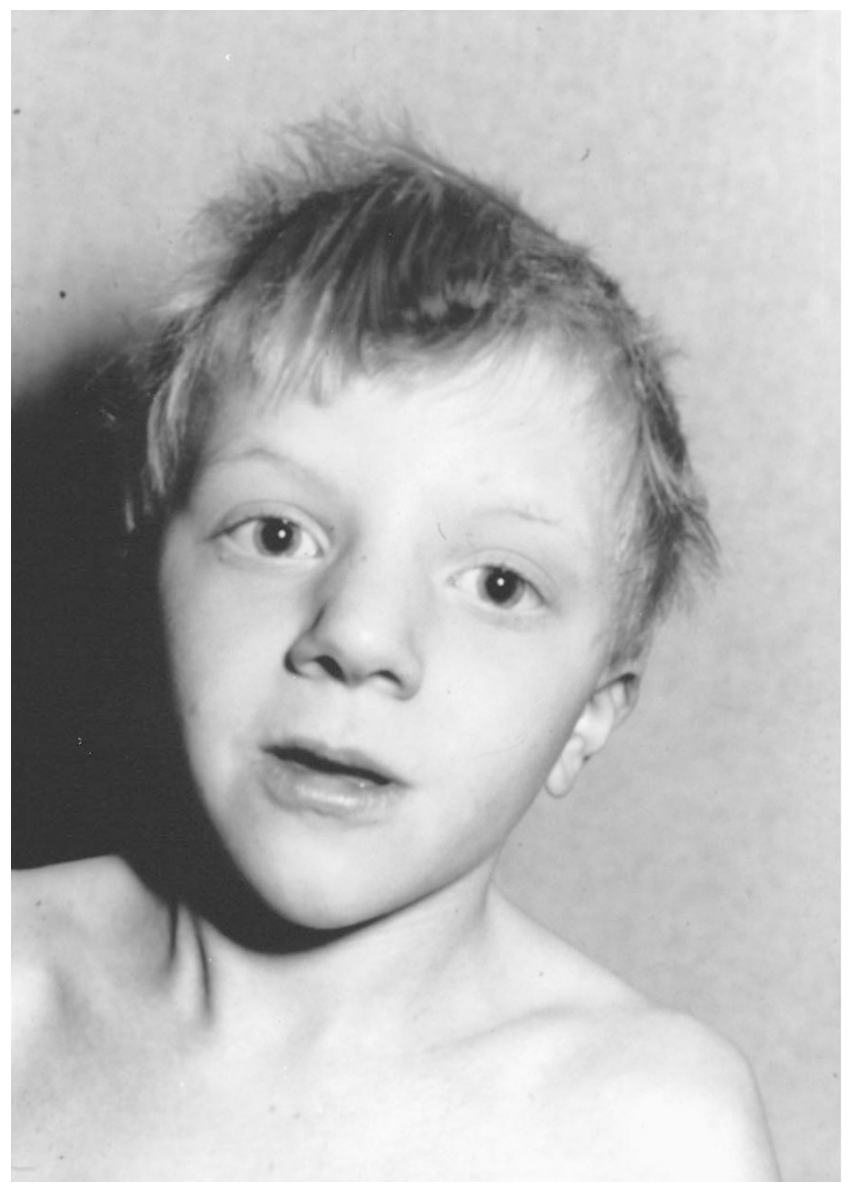

reference sample, ${ }^{9}$ if $\mathrm{CEPH}$ data for that particular marker were available.

Two-point lod scores were calculated using the FA ST L IN K version of the M LINK and ILINK computer programs, ${ }^{10}$ with penetrance set at 1.0 for heterozygotes and a gene frequency of 0.0002 for CFC/NS. A llele frequencies for the different markers in Caucasian populations were obtained from GD B.

\section{Results}

Linkage analysis in this NS/CFC family, without inclusion of the two individuals diagnosed with CFC syndrome, results in a maximum lod score of 3.832 at zero recombination for marker D 12584 (Table 1). This means that the NS phenotype in this family links to $12 q 24$.

Linkage analysis including the two individuals with CFC syndrome showed convincing evidence for linkage between NS/CFC and the $12 q 24$ dinucleotide repeat markers. The dinucleotide repeat markers in Table 1 are

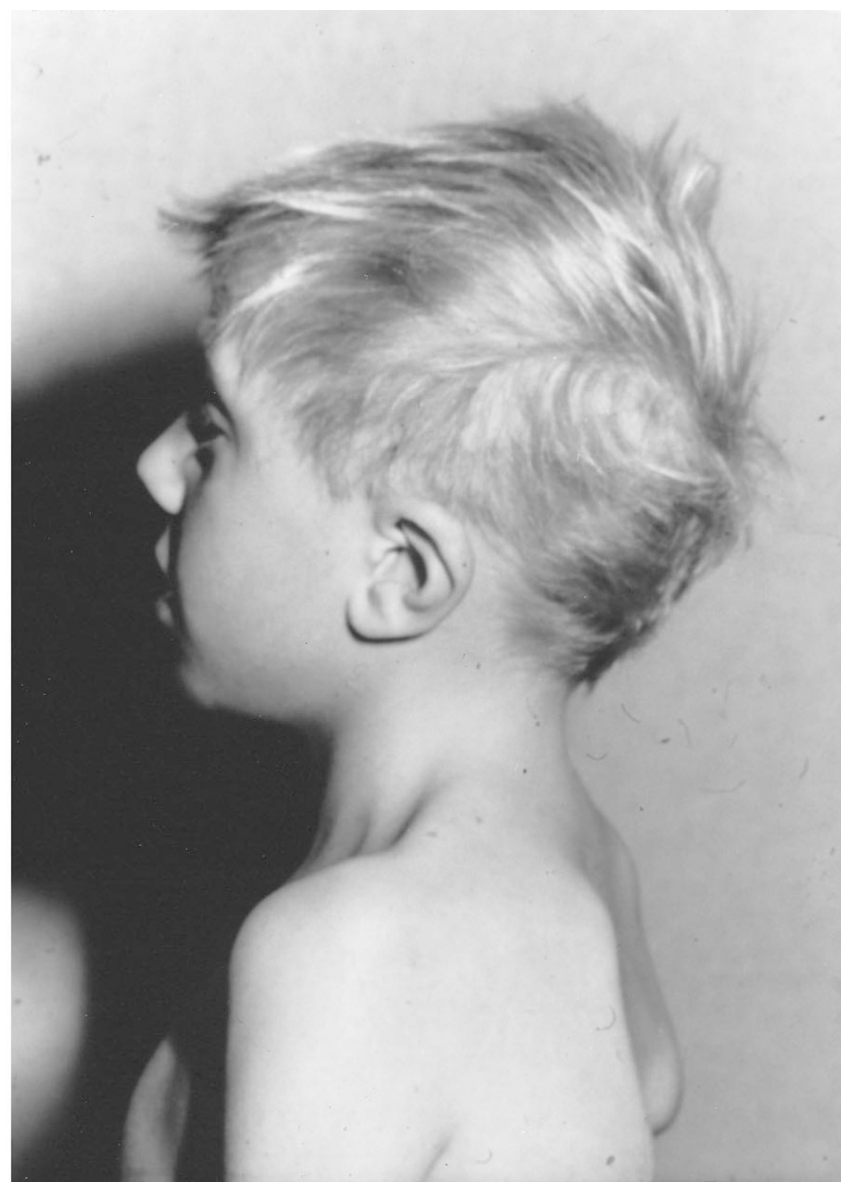

Figure 2 Face and profile of individual IV 1 diagnosed with CFC syndrome. N ote the sparse scalp hair, scanty eyebrows and dry eczematous skin 

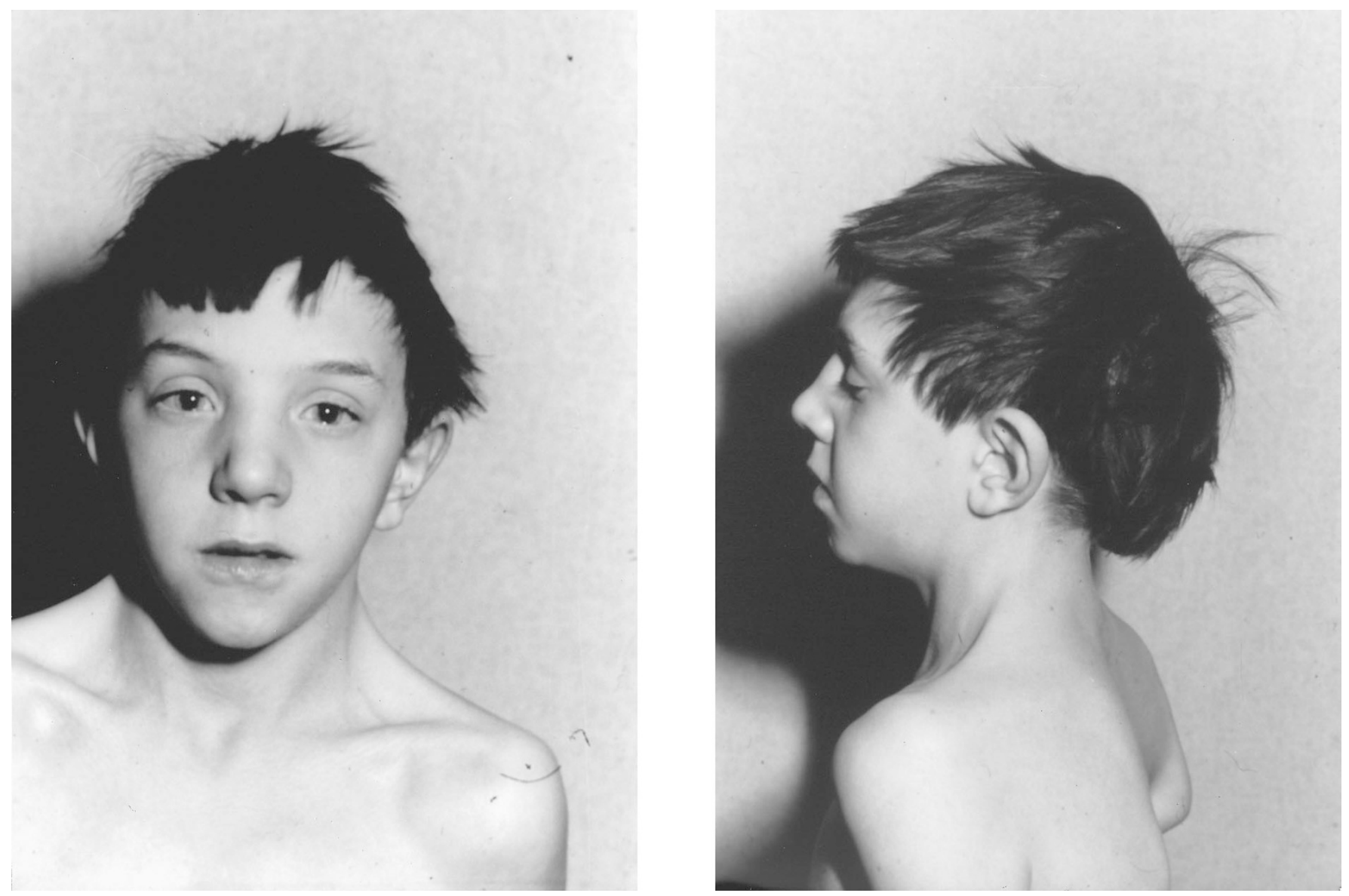

Figure 3 Face and profile of individual IV2, diagnosed with CFC syndrome. Note the same facial characteristics as his brother (Figure 2). This individual also shows a marked ptosis of the eyelids and a webbed neck.

Table 1 Two-point lod score table of NS/CFC syndrome versus 12q24 markers

\begin{tabular}{|c|c|c|c|c|c|c|c|c|c|}
\hline \multirow[b]{2}{*}{ Locus } & \multicolumn{9}{|c|}{ L od score at $\theta$} \\
\hline & 0.00 & 0.01 & 0.05 & 0.10 & 0.20 & 0.30 & 0.40 & $Z \max$ & $\theta \max$ \\
\hline D 12S1613 & $-\infty$ & 1.263 & 1.765 & 1.808 & 1.546 & 1.087 & 0.510 & 1.816 & 0.085 \\
\hline D 12584 & 4.434 & 4.360 & 4.055 & 3.656 & 2.787 & 1.807 & 0.734 & 4.434 & 0.000 \\
\hline D 12S84-CFC & 3.832 & 3.767 & 3.498 & 3.146 & 2.379 & 1.515 & 0.576 & 3.832 & 0.000 \\
\hline D $12 S 105$ & 0.440 & 0.438 & 0.429 & 0.410 & 0.353 & 0.268 & 0.152 & 0.440 & 0.000 \\
\hline D $12 S 1583$ & 2.709 & 2.666 & 2.486 & 2.252 & 1.740 & 1.161 & 0.507 & 2.709 & 0.000 \\
\hline D 12S1339 & 3.311 & 3.259 & 3.044 & 2.762 & 2.148 & 1.453 & 0.666 & 3.311 & 0.000 \\
\hline D 125809 & 3.311 & 3.259 & 3.044 & 2.762 & 2.148 & 1.453 & 0.666 & 3.311 & 0.000 \\
\hline D $12 S 129$ & 1.505 & 1.475 & 1.349 & 1.185 & 0.834 & 0.462 & 0.133 & 1.505 & 0.000 \\
\hline D 12S1341 & $-\infty$ & 1.263 & 1.765 & 1.808 & 1.546 & 1.087 & 0.510 & 1.816 & 0.085 \\
\hline D 12S354 & 0.602 & 0.593 & 0.558 & 0.511 & 0.408 & 0.292 & 0.158 & 0.602 & 0.000 \\
\hline D 12S1023 & 1.204 & 1.187 & 1.115 & 1.021 & 0.816 & 0.585 & 0.317 & 1.204 & 0.000 \\
\hline D 12S369 & $-\infty$ & 1.263 & 1.765 & 1.808 & 1.546 & 1.087 & 0.510 & 1.816 & 0.085 \\
\hline D $12 S 79$ & $-\infty$ & 2.747 & 3.159 & 3.084 & 2.567 & 1.821 & 0.923 & 3.165 & 0.060 \\
\hline D $12 S 366$ & $-\infty$ & 2.153 & 2.602 & 2.574 & 2.159 & 1.529 & 0.764 & 2.618 & 0.066 \\
\hline
\end{tabular}

Two-point lod score results obtained in the reported family, at different recombination frequencies ( $\theta)$ and with inclusion of the two individuals with CFC syndrome. D ata in row D 12S84-CFC refer to the results obtained with marker D 12584 without including the two individuals with CFC syndrome. 
ordered from the centromere to the telomere according to data from the Third International Workshop on H uman Chromosome $12 \mathrm{M}$ apping $1995 .{ }^{11}$ Three markers (D 12S84, D 12S1339 and D 12S809) showed a maximum lod score of more than 3 at no recombination and 1 (D 12S79) at 6\% recombination. The highest twopoint lod score was obtained with marker D12S84 $(4.434 ; \theta=0)$ (Table 1$)$.

A crossover in individual $11 / 2$ between NS/CFC and markers D 12S1341, D 12S369, D 12S79 and D 12S366 was observed. M arkers D 12S354 and D 12S1023 were not informative in individual III2. This crossover indicates that the NS/CFC locus is proximal to D 12S1341 (Figure 1 and 4). Markers D 125809 and D 12S129, proximal to the crossover, and marker D 12S1341, distal to the crossover have been mapped to the same yeast artificial chromosome (YAC) (920g2). ${ }^{12}$ The exact position of D $12 S 129$ in relation to $D 12 S 809$ is not known. ${ }^{11}$ No other markers were available for a more precise mapping of the crossover.

A nother crossover was observed in an unaffected individual (IIII) between the NS/CFC locus and markers D 12S78, D 12S330 and D 12S1613. This indicates that the NS/CFC in this family is localized between marker D12S1613 proximally and marker D 12S1341 distally (Figures 1 and 4). A multipoint linkage analysis using markers D 12S330, D 12 S84 and D 12S1341 showed a maximum lod score of 4.43 at D 12S84. This family shows linkage to exactly the same region on the long arm of chromosome 12 as NS. ${ }^{2}$

A remarkable finding is the presence of three pairs of dizygotic twins in the offspring of two affected females. In the previously reported NS family (2) there is also a dizygotic twin in the offspring of an affected female. In these two families four dizygous twins are present on a total of 27 full term pregnancies in seven affected females. The frequency of dizygotic twinning in these

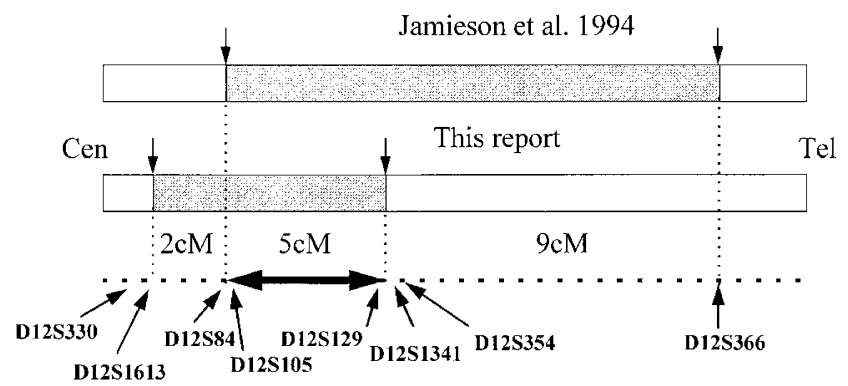

Figure 4 Schematic of the genetic map showing the localization of several markers used in the linkage study. C rossovers in the present family and in the previous reported family with NS2 are indicated by vertical arrows ( $\downarrow)$ families is markedly higher than expected ${ }^{13}$ for this region of Europe $(R R=27.4$; Fisher exact test: $p<0.001)$.

\section{Discussion}

The family reported here is the largest family in the literature with NS/CFC. L inkage analysis unequivocally maps the NS/CFC phenotype in this family to the same region on chromosome $12 \mathrm{q}$ as $\mathrm{NS}{ }^{2}{ }^{2} \mathrm{E}$ ven if the two individuals with CFC syndrome are excluded from the analysis the maximum lod score remains higher than 3 for D 12S84. This is the second family in the literature with proven linkage to 12 q24. A crossover is described between NS/CFC and markers D 12S1341, D 12S369, D $12 S 79$ and D 12S366, and with markers located proximally to D $12 S 84$ (D 12S78, D 12S330, D 12S1613). The NS/CFC phenotype in this family is localized in a $7 \mathrm{cM}$ region between markers $\mathrm{D} 12 \mathrm{~S} 1613$ proximally and D 12S1341 distally (Figure 4). A three-generation NS family previously reported showed a recombination between NS and markers D12S84 proximally and D 12S366 distally, mapping NS to a $14 \mathrm{CM}$ region between these two markers ${ }^{2}$ A ssuming that NS and NS/CFC are caused by a defect in the same gene, we can narrow the candidate gene region for NS/CFC to a $5 \mathrm{CM}$ interval between D 12S84 and D 12S1341 (Figure $4)$.

In the reported family the linkage data and the clinical features (NS in some, CFC syndrome in others) support the hypothesis that CFC syndrome is a variant of NS. M ost CFC cases are sporadic, probably because these individuals are so severely affected that they do not reproduce. It is possible that in some families CFC syndrome is not related to a gene on $12 q 24$, but this has not yet been documented. However, present and previous data indicate that in several cases CFC syndrome is a severe expression of $\mathrm{NS}^{5-7}$ We hypothesize that CFC syndrome is related to NS, either as an allelic variant or as a disorder with a similar molecular and cellular pathogenic mechanism.

Several reports suggested the possibility of CFC syndrome and NS being contiguous gene syndromes. ${ }^{6,7}$ NS and CFC syndrome can result from different mutations or deletions in the same $5 \mathrm{cM}$ chromosomal region in 12q24. In the reported family, however, the variable expression of the phenotype resulted in NS in some individuals and CFC syndrome in others. M oreover, a contiguous gene syndrome results from a deletion 
of several genes but none of the 7 markers in the $5 \mathrm{~cm}$ candidate gene region showed any evidence for a deletion in this family. Nor did $\mathrm{K}$ remer et al. observe any abnormalities in a systematic screen with 28 12q24 polymorphic markers in 10 unrelated CFC syndrome and $100 \mathrm{NS}$ individuals. ${ }^{14} \mathrm{~A}$ contiguous gene syndrome cannot be ruled out by these data, but it is less likely.

A remarkable finding in the reported family is the presence of three dizygotic twins in the offspring of two affected females (Figure 1). A dizygotic twin in the offspring of an affected female is also observed in the other large NS family with linkage to $12 q 24 .^{2}$ It is possible that an increased frequency of dizygotic twinning is associated with NS/CFC linked to $12 q 24$. The fragile $X$ syndrome is another example of a $M$ endelian disorder with an increased frequency of dizygotic twinning. ${ }^{15,16}$ It is also possible that the association between NS/CFC and twinning is fortuitous because of the large number of offspring. H owever we believe the possible association merits further exploration.

L arge families such as the one reported here are very helpful for fine mapping and cloning of the NS/CFC gene. In the presence of genetic heterogeneity, smaller families with NS/CFC are not very informative for linkage analysis, and their use in defining the candidate gene region is limited. However these smaller families might shed some light on the possible association with dizygotic twinning.

\section{Acknowledgements}

We thank $\mathrm{H}$ annie $\mathrm{K}$ remer, Nijmegen, for the PCR-primers for markers D12S1605, D 12S105, D 12S1583, D 12S1339, D 12S1343, D 12S809, D 12S129, D 12S1341，D 12S1023. We thank Elly Pijkels for exploring the pedigree and for the collection of blood samples. Professor Marc Gewillig is acknowledged for providing the clinical data on the cardiac abnormalities in individuals III 2 and IV 4.

\section{References}

1 A llanson JE: Noonan syndrome. J Med Genet 1987; 24:9-13.

2 Jamieson $C R$ et al: $M$ apping a gene for N oonan syndrome to the long arm of chromosome 12. Nature G enet 1994; 8:357-360.

3 Reynolds J F et al: New multiple congenital anomalies/ mental retardation syndrome with cardio-facio-cutaneous involvement - the CFC syndrome. A m J M ed G enet 1986; 25: 413-427.

4 Neri G, Zollino $M$ : More on the Noonan-CFC controversy. A m J M ed G enet 1996; 65: 100.

5 L eichtman LG : A re cadio-facio-cutaneous syndrome and Noonan syndrome distinct? A case of CFC offspring of a mother with Noonan syndrome. Clin Dysmorph 1996; 5: 61-64.

6 Fryer $\mathrm{AE}, \mathrm{H}$ olt PJ, Hughes $\mathrm{HE}$ : The cardio-facio-cutaneous (CFC) syndrome and Noonan syndrome: are they the same?. A m J M ed G enet 1991; 38: 548-551.

7 L orenzetti M E, Fryns J P: R etinitis pigmentosa in a young man with Noonan syndrome: further evidence that $\mathrm{Noo-}$ nan syndrome (NS) and the cardio-facio-cutaneous syndrome (CFC) are variable manifestations of the same entity. A m J M ed G enet 1996; 65: 97-99.

8 Fryns J P, Volcke P, Van D en B erghe $\mathrm{H}$ : The cardio-faciocutaneous (CFC) syndrome: autosomal dominant inheritance in a large family. G enet Counsel 1992; 3: 19-24.

9 G yapay Get al: The 1993-94 G énéthon human genetic linkage map. Nature G enet 1994; 7 (Suppl. 246).

10 Cottingham RW, I dury R M, Schäffer A A : Faster sequential genetic linkage analysis. A m J Hum Genet 1993; 53: 252-263.

11 Marynen P, Kucherlapati R: Report of the third international workshop on human chromosome 12 mapping 1995. Cytogenet Cell G enet 1996; 73: 1-24.

12 Chumakov I et al: A YAC contig map of the human genome. Nature 1995; 377 (6547 Suppl.): 174-297.

13 Vlietinck $R$ et al: The validity of Weinberg's rule in the East Flanders Prospective Twin Survey (EFPTS). A cta G enet M ed Gemellol Roma 1988; 37: 137-141.

$14 \mathrm{~K}$ remer $\mathrm{H}$ et al: A search for (micro)deletions in CFC syndrome and Noonan syndrome patients. Am J Hum Genet 1996; 59: A 267.

15 Partington M W, M oore DY, Turner G M : Confirmation of early menopause in fragile $X$ carriers. A m J Med Genet 1996; 64: 370-372.

16 Schwartz CE et al: Obstetrical and gynecological complications in fragile $X$ carriers: a multicenter study. A m J M ed $\mathrm{G}$ enet 1994; 51: 400-42. 